\title{
Thomas Haffner \\ Konrad Haebler und die Entwicklung vom lokalen zum internationalen Inkunabelkatalog
}

Neben einem Bücherregal, zurückgelehnt in einen Sessel, den rechten Unterarm neben zwei Büchern aufgelegt, ein leicht geöffnetes Buch in der Hand, bekleidet mit weit geöffnetem Jackett, Weste, blaugrauer Krawatte und weißem Stehkragen, blickt ein älterer Herr mit Stirnglatze und Knebelbart dem Betrachter gestreng unter Schlupflidern durch einen goldenen Zwicker auf einer langen spitzen Nase entgegen - der 71-jährige Historiker, Bibliothekar, Inkunabel- und Einbandforscher Professor Dr. Konrad Haebler auf einem Ölgemälde des Dresdner Malers Walther Günther Julian Witting (1864-1940) aus dem Jahr 1928. ${ }^{1}$

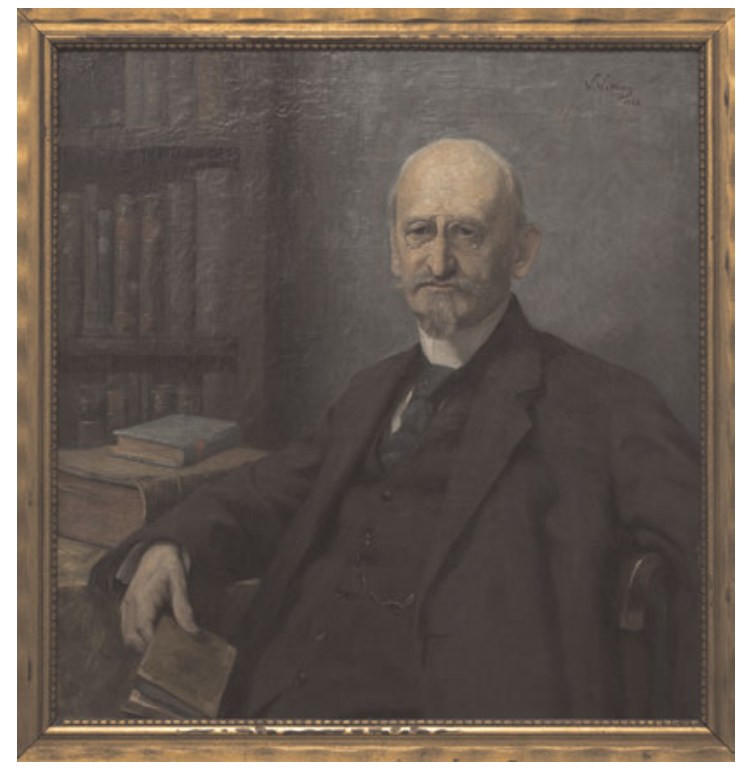

Abb. 1: Walther Günther Julian Witting: Bildnis Konrad Haebler (1928), Öl auf Leinwand. SLUB Dresden/Deutsche Fotothek, Henrik Ahlers.

1 Es befindet sich im Besitz der SLUB Dresden. Ein weiteres, ebenfalls von Witting gemaltes Porträt aus dem Jahr 1926 in der Staatsbibliothek Preußischer Kulturbesitz in Berlin zeigt Haebler mit der Lupe über eine Inkunabel gebeugt.

ว Open Access. () 2018 Thomas Haffner, publiziert von De Gruyter. (๔) BY-Nc-ND Dieses Werk ist lizenziert unter der Creative Commons Attribution-NonCommercial-NoDerivatives 4.0 Lizenz.

https://doi.org/10.1515/9783110587524-036 
Die künstlerische Darstellung entspricht der Charakterisierung Haeblers im Nachruf seines Kollegen Wieland Schmidt im Zentralblatt für Bibliothekswesen: „Wie er streng gegen sich war, war er es auch gegen seine Untergebenen und Mitarbeiter. Für persönliche Gefühle blieb in diesem Leben wenig Raum. Stets wahrte er die Distanz, und eigentlich nahegekommen ist ihm niemand." 2 Andererseits schwärmte Haeblers wissenschaftliche Hilfsarbeiterin und spätere Einbandforscherin Ilse Schunke, „mit wieviel gütigem Anteil und gutem Rat“ Haeblers Briefe sie in den Jahren des heraufziehenden Nationalsozialismus begleitet hätten und wie „wunderbar [...] packend und anschaulich“ er habe erzählen können. ${ }^{3}$

Konrad Haebler wurde am 29. Oktober 1857 in Dresden als Sohn des Lehrers, Dichters und Privatgelehrten Carl Gotthelf Haebler geboren. Nach dem Abitur an der Dresdner Kreuzschule nahm er an Ostern 1876 ein Studium der Geschichte und Philosophie an der Universität Leipzig auf. Noch bevor er am 20. Oktober 1880 das Examen für die Kandidatur zum höheren Schulamt erfolgreich abgelegt hatte, trat er am 1. November 1879 eine Stelle als wissenschaftlicher Hilfsarbeiter an der Königlichen Öffentlichen Bibliothek in Dresden an. 1881 avancierte er zum Kustos (Bibliothekssekretär) und wurde im darauf folgenden Jahr an der Universität Leipzig mit der Arbeit Der Streit Ferdinand's des Katholischen und Philipp's I. um die Regierung von Castilien 1504-1506 promoviert. ${ }^{4}$ Auf dem Gebiet der Geschichte der iberischen Halbinsel forschte und publizierte Haebler auch weiterhin erfolgreich neben seinen Pflichten in der Bibliothek, die ihm „reichlich Muße“ zur Pflege seiner „spanischen Liebhaberei“ ließ. ${ }^{5}$ Worin

2 Schmidt, Wieland: Konrad Haebler. In: Zentralblatt für Bibliothekswesen 64 (1950) H. 11-12. S. 403-413, hier S. 412.

3 Schunke, Ilse: Erinnerungen an Konrad Haebler. In: Über Bücher, Bibliotheken und Leser. Gesammelte Beiträge zum 60. Geburtstag von Horst Kunze. Hrsg. von Werner Dube, Othmar Feyl u. Gotthard Rückl. Leipzig: Bibliographisches Institut 1969. S. 185-192, hier S. 188 u. 191. 4 Gedruckt 1882 in Dresden durch die Albanus'sche Buchdruckerei; mit Kurzbiografie auf S. 134. Vgl. auch Nitzschke, Katrin: Haebler, Konrad. In: Dresdner Bibliothekarinnen und Bibliothekare. Hrsg. von Martina Schattkowsky, Konstantin Hermann u. Roman Rabe. Leipzig: Leipziger Universitätsverlag 2014. S. 107. http://saebi.isgv.de/biografie/Konrad_Haebler_(18571946). Alle aufgeführten Internetressourcen wurden zuletzt am 27. Dezember 2017 aufgerufen. 5 Haebler, Konrad: Vom spanischen Haebler. Von ihm selbst erzählt. In: Die Bibliothek Geh. Rat Prof. Dr. Konrad Haebler. Nestor der deutschen Inkunabelkunde. Bibliographie - Literaturwissen, Inkunabelkunde, Buch- und Schriftwesen. Im Anhang: Hispanica, alte Drucke. Dresden: v. Zahn \& Jaensch 1943. S. 1-3, hier S. 1. Vgl. auch Boddin, Heinz: Konrad Haeblers Arbeiten zur spanischen Geschichte und zum Frühdruck in Spanien. In: Dresden und Spanien. Akten des interdisziplinären Kolloquiums, Dresden, 22.-23. Juni 1998. Hrsg. von Christoph Rodiek. Frankfurt am Main: Vervuert 2000. S. 125-133; Torres Santo Domingo, Marta: Konrad Haebler y España en el Archivo del Gesamtkatalog der Wiegendrucke. In: Pecia Complutense 14 (2017) H. 26. S. 70-90. http://biblioteca.ucm.es/BUCM/pecia/58231.php. Im Laufe seines 
seine Amtsgeschäfte bestanden, geht aus den handschriftlichen Jahresberichten hervor, die Haebler gleich seinen Amtskollegen seit 1888 dem im Jahr zuvor angetretenen Bibliotheksdirektor Franz Schnorr von Carolsfeld vorzulegen hatte. ${ }^{6}$ $\mathrm{Zu}$ seinen Routinetätigkeiten gehörten Katalogisierung, Einrangieren (d. h. Vergabe systematischer Signaturen), Expedieren (d. h. Ausheben) und Einstellen von Büchern. Darüber hinaus war er immer wieder mit Arbeiten am neuen alphabetischen Kapselkatalog, der den seit 1796 geführten alphabetischen Bandkatalog ablöste, beschäftigt, sei es, dass er den Bestand diverser Fachgruppen alphabetisch ordnete, sei es, dass er Signaturen eintrug oder redaktionelle Arbeiten verrichtete. 1890 fertigte er einen Standortkatalog für den Handbestand im Lesesaal und arbeitete Bücher aus dem Nachlass des Schweizer Hispanisten Edmund Dorer ein, der am 5. Mai 1890 in Dresden verstorben war. 1891 war er für die Umsetzung des Ende Mai dieses Jahres vollendeten neuen alphabetischen Katalogs in den Katalogsaal in der ersten Etage des Bibliotheksgebäudes, d. h. des Japanischen Palais verantwortlich. 1893 erstellte er ein Verzeichnis über den so genannten Maya-Apparat, der vom ehemaligen Bibliotheksdirektor Ernst Förstemann gesammelten Literatur und Korrespondenz zur Erforschung und Entzifferung der Dresdner Maya-Handschrift. ${ }^{7} 1895$ bis 1898 verzeichnete er Bücher aus der 1884 übernommenen Bibliothek der Herzöge von BraunschweigOels im alphabetischen Katalog. Am 1. Januar 1896 wurde Haebler vom Kustos zum Bibliothekar befördert und erhielt am 23. April, ${ }^{8}$ zum Geburtstag des Königs Albert von Sachsen, den Titel Professor, sodass er seine Jahresberichte fortan als „Prof. Dr. K. Haebler, Bibliothekar“ unterzeichnen konnte.

Im selben Jahr begann er, sich mit dem Dresdner Inkunabelbestand zu befassen, indem er einen „Auszug des Incunabeln-Kataloges“ für Marie Pellechet (1840-1900) erstellte, die dabei war, sämtliche Inkunabeln in den öffentlichen

\footnotetext{
Lebens brachte es Haebler auf insgesamt 330 Veröffentlichungen (vgl. Schmidt, Haebler (wie Anm. 2), S. 405; Die Schriften Konrad Haeblers. Berlin: Kommission für den Gesamtkatalog der Wiegendrucke 1937 [303 Nummern]).

6 Erhalten sind Haeblers Berichte für die Jahre 1888, 1890-1896 und 1898-1906 (SLUB, Bibl. Arch.III.G,Vol.647). Für 1889 und für 1897 fertigte Haebler wahrscheinlich keine Berichte an, weil er auf längeren Reisen war: Einmal begleitete er als Dolmetscher den Prinzen Friedrich August von Sachsen auf einer Rundreise durch Spanien, das andere Mal war er auf eigene Rechnung zu Archivrecherchen in Spanien und Portugal unterwegs.

7 Vgl. Haebler, Konrad: Die Maya-Litteratur und der Maya-Apparat zu Dresden. In: Centralblatt für Bibliothekswesen 12 (1895) H. 12. S. 537-575. Haeblers inhaltliches Interesse an der MayaForschung spiegeln einige Briefe von ihm an Förstemann zwischen 1894 und 1904 (Mscr.Dresd. e.200,I,1,Nr.171-194).

8 Schmidt, Haebler (wie Anm. 2), S. 404 gibt als Datum den 7. April 1896 an.
} 
Bibliotheken Frankreichs aufzunehmen. ${ }^{9}$ Das erste handschriftliche Verzeichnis der Dresdner Inkunabeln datiert vom November $1818^{10}$ und stammt von Friedrich Adolf Ebert, seit 1814 Sekretär und ab 1828 bis zu seinem tödlichen Sturz von der Bücherleiter 1834 Oberbibliothekar der Königlichen Öffentlichen Bibliothek. ${ }^{11}$ Darin sind 1553 Drucke mit den Signaturen des allgemeinen Druckschriftenbestands nach Druckjahren geordnet und mit Nachweisen in den Annales typographici $^{12}$ des ersten eigentlichen Inkunabel- und Frühdruckbibliografen Georg Wolfgang Panzer (1729-1805) versehen. In diesem Zusammenhang sei angemerkt, dass Ebert am 24. März und am 1. Mai 1819 beim „Bibliographisiren“ das seltene Glück hatte, zwei als Einbandmaterial verwendete Fragmente der wie er sofort erkannte - 42-zeiligen Gutenberg-Bibel zu finden. ${ }^{13}$ Haebler stützte sich bei seiner Zuarbeit für Pellechet auf ein zweites handschriftliches Verzeichnis der Dresdner Inkunabeln, ${ }^{14}$ das der Bibliothekar und Bibliograf Ernst Gustav Vogel (1797-1874) ${ }^{15}$ in den Jahren 1866 bis 1870 zusammengestellt hatte. Es enthält sehr knappe Titelaufnahmen von rund 2000 Drucken in drei Abteilungen, ${ }^{16}$ die in sich alphabetisch nach Verfassernamen geordnet sind, und Verweise auf Exemplarbeschreibungen in Johann Christian Goetzes Merckwürdigkeiten Der Königlichen Bibliotheck zu Dreßden (1743-1748) sowie auf jüngere Literatur wie das 1826-1838 erschienene einschlägige Repertorium bibliographi-

9 Pellechet, Marie u. Marie-Louis Polain (Hrsg.): Catalogue général des incunables des bibliothèques publiques de France. Bd. 1-3. Paris: Picard 1897-1909; Faksimile des Manuskripts für Bd. 4-26. Nendeln, Liechtenstein: Kraus-Thomson 1970.

10 Index librorum seculo XV impressorum quos servat Bibliotheca Regia Dresdensis (Bibl. Arch.I.Ba,Vol.188.b).

11 Vgl. Mühlner, Manfred: Ebert, Friedrich Adolf. In: Dresdner Bibliothekarinnen und Bibliothekare (wie Anm. 4). S. 89-90. http://saebi.isgv.de/biografie/Friedrich_Adolf_Ebert_(17911834).

12 Panzer, Georg Wolfgang: Annales typographici. Bd. 1-5 (Ab artis inventae origine ad annvm MD). Nürnberg: Zeh 1793-1797.

13 Vgl. Ebert, Friedrich Adolf: Diarium über meine Arbeiten auf der Akademischen Bibliothek zu Leipzig und der Königlichen Bibliothek zu Dresden 1813-1822. Dresden: Sächsische Landesbibliothek 1990, S. 112-113.

14 Verzeichnis der Incunabeln der königl. Öffentlichen Bibliothek zu Dresden (SLUB, Bibl. Arch.II.Ea,Vol.461).

15 Vgl. Hermann, Konstantin: Vogel, Ernst Gustav. In: Dresdner Bibliothekarinnen und Bibliothekare (wie Anm. 4). S. 185-186. http://saebi.isgv.de/biografie/Ernst_Gustav_Vogel_(17971874).

16 I. Xylographische Werke und Fragmente, II. Incunabeln im engeren Sinne und in alphabetischer Ordnung, III. Undatirte Drucke. 
cum von Ludwig Hain ${ }^{17}$ oder die von Jan Willem Holtrop 1868 herausgegebene lithografische Beispielsammlung aus niederländischen Bibliotheken. ${ }^{18}$ Die Signaturen des allgemeinen Druckschriftenbestands wurden in einer eigenen Spalte mit Bleistift ergänzt. Hie und da finden sich handschriftliche Bemerkungen von Haebler, der die fachliche Qualität des Katalogs bemängelte. ${ }^{19}$

Schon 1894 hatte Haebler entsprechend seinem hispanistischen Interesse einen Aufsatz über Deutsche Buchdrucker in Spanien und Portugal veröffentlicht, ${ }^{20}$ der 1897 in erweiterter Form als Monografie in englischer Sprache erschien. ${ }^{21}$ Nach der Rückkehr von einer Studienreise nach Spanien und Portugal von November 1897 bis Februar 1898 erbat sich Haebler „dienstlich den Auftrag, den recht beträchtlichen Bestand der Dresdner Inkunabeln unter Berücksichtigung der modernsten Forschungsmethoden zu katalogisieren “. ${ }^{22}$ Darunter verstand Haebler die vom englischen Bibliothekar Robert Proctor (1868-1903) bei der Katalogisierung der Inkunabeln des British Museum in London und der Bodleian Library in Oxford angewandte exakte Bestimmung der Drucke durch Vermessung der Kegelhöhe der Typen (Lettern) auf einer einheitlichen Basis von 20 Zeilen und durch Charakterisierung der von den einzelnen Druckern verwendeten Typenformen. ${ }^{23}$ Wie einst Panzer ging Haebler bei seiner Katalogisierungsarbeit von den Druckorten und Druckern aus.

17 Hain, Ludwig: Repertorium bibliographicum. 4 Teile in 2 Bdn. Stuttgartiae et Tubingae: Cotta $1826-1838$.

18 Monuments typographiques des Pays-Bas au quinzième siècle. Collection de fac-simile d'après les origineaux conservés à la Bibliothèque royale de La Haye et ailleurs. La Haye: Nijhoff 1868.

19 Vgl. Haebler, Konrad: Die Inkunabelsammlung der Sächsischen Landesbibliothek zu Dresden. In: Minerva-Zeitschrift 3 (1927) H. 6-7. S. 138-145, hier S. 145.

20 Haebler, Konrad: Deutsche Buchdrucker in Spanien und Portugal. In: Centralblatt für Bibliothekswesen 11 (1894) H. 12. S. 529-564.

21 Haebler, Konrad: The early printers of Spain and Portugal. London: Chiswick Press 1897.

22 Haebler, Konrad: Wie ich Inkunabelforscher wurde. Ein Stückchen Lebensgeschichte. St. Gallen: Tschudy 1931, o. P.

23 Vgl. Proctor, Robert: An index to the early printed books in the British Museum. Teil 1. London: Paul, Trench, Trübner 1898; Haebler, Konrad: Handbuch der Inkunabelkunde. Leipzig: Hiersemann 1925, S. 24-27. 


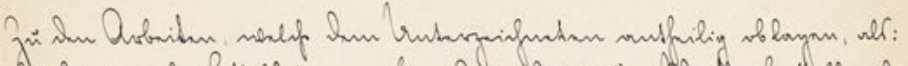

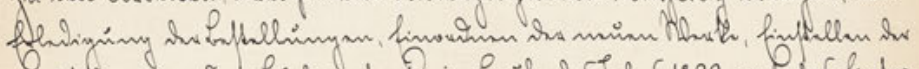

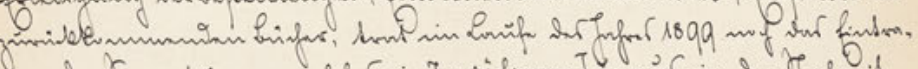

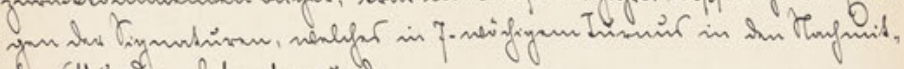

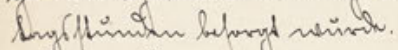

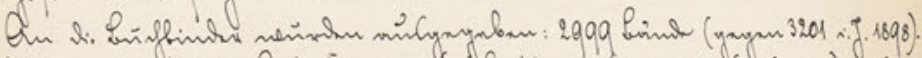

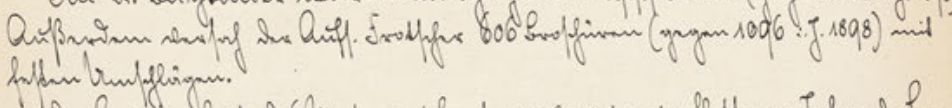

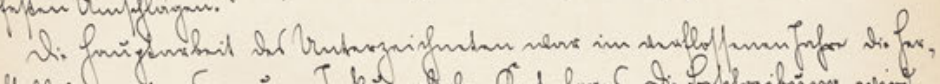

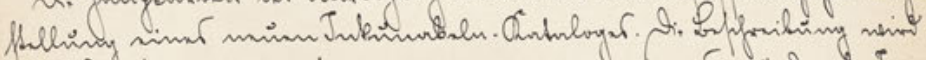

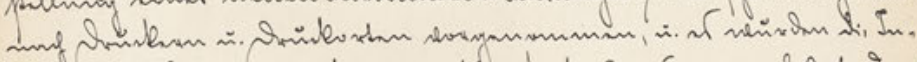

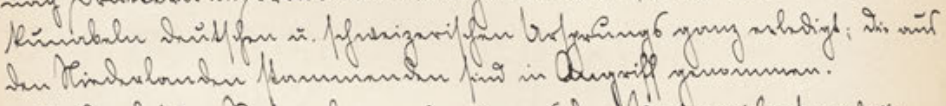

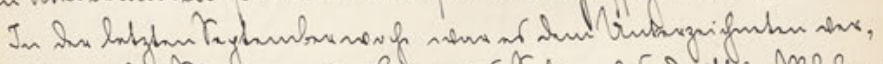

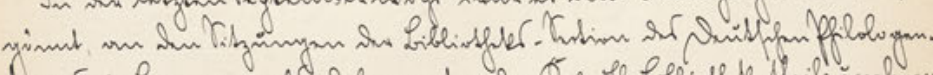

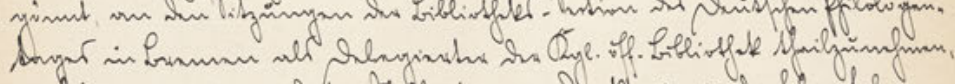

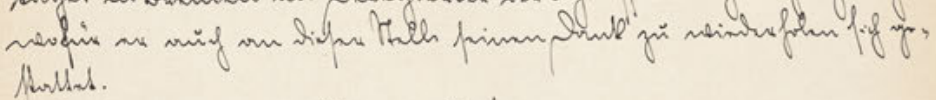

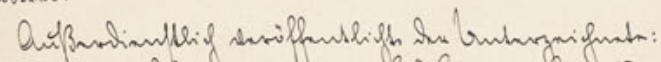

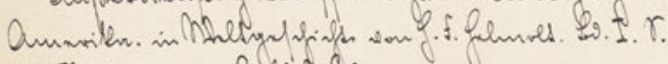

Tter Tbericum. in lisbe f. Libl.

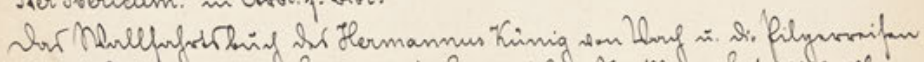

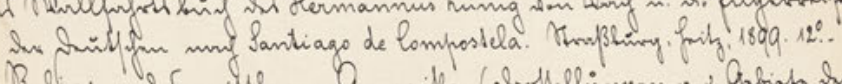

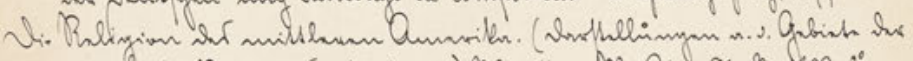

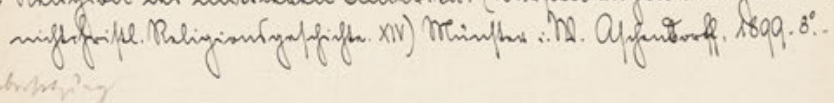

$$
\begin{gathered}
\text { Grof. Dr. K. Hacbler } \\
\text { Liblirfflems. }
\end{gathered}
$$

Abb. 2: Konrad Haebler: Jahresbericht 1899. SLUB Dresden, Bibl.Arch.III.G,Vol.647. SLUB Dresden/Deutsche Fotothek. 
Seinen Jahresberichten zufolge schloss er 1899 die Beschreibung der deutschen und schweizerischen Drucke ab und begann mit der Beschreibung der niederländischen Drucke. 1900 beschrieb er den Rest der niederländischen Drucke, die französischen Drucke und die Drucke aus den italienischen Städten von A bis Ve. 1901 folgten die restlichen italienischen Drucke und der größte Teil der Drucke ohne Druckvermerk sowie vorläufige Register nach Druckorten und Druckern. Nach einigen Nachträgen und Ergänzungen war der Katalog 1902 fertiggestellt. Haebler beschrieb 2093 Inkunabeln auf Einzelblättern, die alphabetisch nach Autoren und Anonyma und darin jeweils chronologisch geordnet, durchnummeriert und in acht patentierten Soennecken-Briefordnern im Quartformat mit goldgeprägten Rückentiteln eingeheftet sind. Hinzu kommen zwei weitere derartige Ordner, die einen dreiteiligen, an Proctor orientierten alphabetisch-chronologischen Index zu Druckorten, Druckern und Drucken in Deutschland, Italien und im übrigen Europa, einen alphabetischen Index der Verfassernamen und anonymen Werktitel, ein Verzeichnis der Vorbesitzer mit rund 600 Einträgen, eine Konkordanz der Katalognummern und der Hain-Nummern und einen Schlüssel zu den Sammelbänden enthalten. Außer den bibliografischen Elementardaten mit Verweis auf die entsprechende Nummer in Hains Repertorium und gegebenenfalls im Supplement von Walter Arthur Copinger ${ }^{24}$ führte Haebler die entsprechenden (später wieder ausgestrichenen) Typennummern aus Proctors Katalog an, notierte Bogensignaturen und Kustoden und machte Angaben zu gedruckten und von Hand hinzugefügten Initialen, zu Wasserzeichen, zu handschriftlichen Einträgen, zu Einbänden und zur Provenienz der Dresdner Exemplare.

24 Copinger, Walter Arthur: Supplement to Hain's Repertorium bibliographicum, or collection towards a new edition of that work, in two parts. London: Sotheran 1895-1902. 


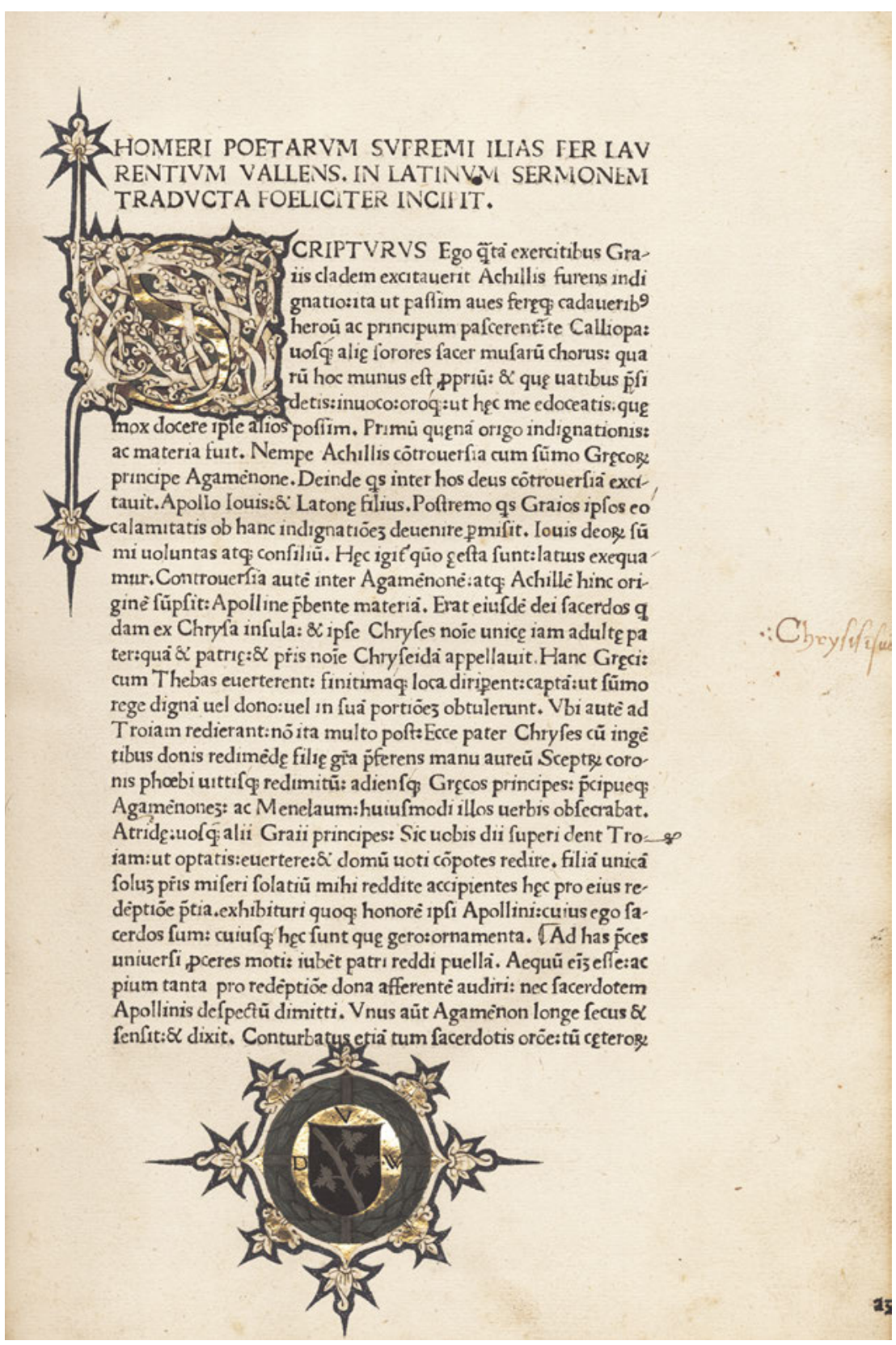

Abb. 3: Homerus: Ilias (Übers.: Laurentius Valla). Brescia: Heinrich 〈von Köln〉, und Statius Gallicus, 24. Nov. 1474. SLUB Dresden, Ink.2134(2), Bl. a3, mit Wappen und Initialen von Dietrich von Werthern. SLUB Dresden/Deutsche Fotothek. 


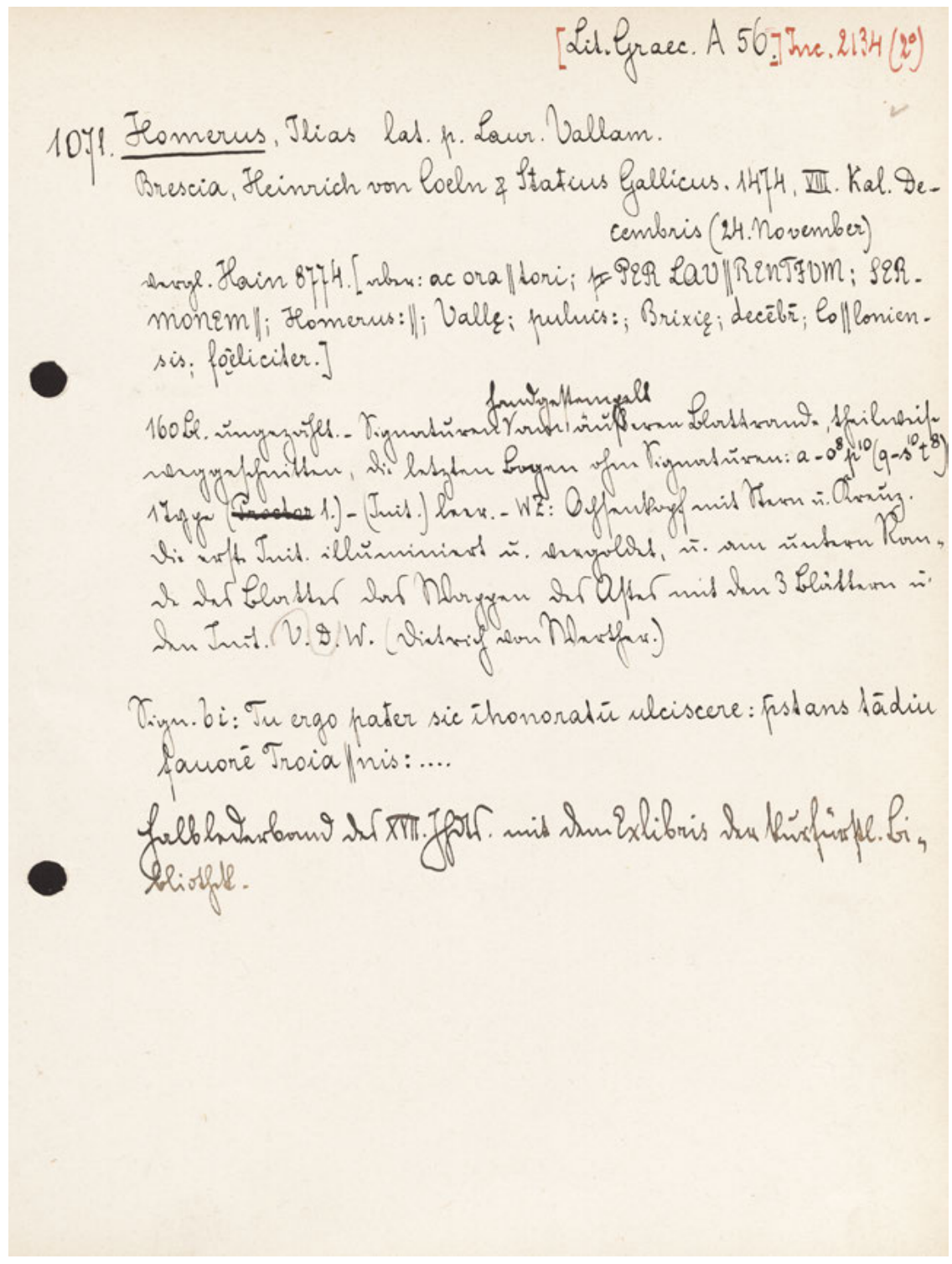

Abb. 4: Konrad Haebler: Beschreibung des Dresdner Exemplars Homerus: Ilias (Übers.: Laurentius Valla). Brescia: Heinrich <von Köln〉, und Statius Gallicus, 24. Nov. 1474, im handschriftlichen Inkunabelkatalog. SLUB Dresden/Deutsche Fotothek.

Mit 496 Drucken überwiegend italienischer Herkunft in 228 Bänden erhielt die Kurfürstliche Bibliothek 1589 durch den Ankauf der umfangreichen Bibliothek 
des Grafen Dietrich von Werthern (1468-1536) und seiner Söhne auf Schloss Beichlingen in Thüringen den größten Inkunabelzugang ihrer Geschichte. ${ }^{25} \mathrm{Im}$ 18. Jahrhundert brachten die Erwerbung mehrerer privater Büchersammlungen, allen voran derer des Zeremonienmeisters Johann von Besser (1654-1729) und des Grafen Heinrich von Bünau (1697-1762), sowie der Aufkauf von sächsischen Schulbibliotheken einen Zuwachs von insgesamt 383 Inkunabeln. Im 19. Jahrhundert kamen 219 Inkunabeln aus der Bibliothek des einstigen Franziskanerklosters in Meißen bzw. aus der Schlossbibliothek in Oels hinzu. ${ }^{26}$

Neben der Inkunabelkatalogisierung und den oben angeführten Routinetätigkeiten war Haebler seinen Jahresberichten zufolge ab 1898 regelmäßig für die Aushändigung von Büchern an die Buchbinder und die Führung des Buchbindejournals zuständig. Außerdem erledigte er einige Arbeiten in der Handschriftensammlung: 1901 glich er die Signaturen der 1839 aus der Sammlung des Missionars Heinrich August Zwick erworbenen tibetischen Handschriften mit dem gedruckten Verzeichnis des Sinologen Berthold Laufer $a b^{27}$ und ließ durch einen Buchbinder „dauerhafte Umschläge“ ${ }^{28}$ für diese Handschriften anfertigen; 1902 verzeichnete er Autografen in den Briefsammlungen von Friedrich Adolf Ebert (Mscr.Dresd.h.23), Johann Gottlob Horn (Mscr.Dresd.b.58), Wilhelm August von Just (Mscr.Dresd.h.38), Karl Preusker (Mscr.Dresd.R.265.d), Eduard Schwender (Mscr.Dresd.h.29) und zwei weitere Autografenkonvolute (Mscr.Dresd.R.52.m und 52.n); 1903 vervollständigte er den Autografenkatalog

25 Vgl. Haffner, Thomas: Illuminierte italienische Inkunabeln und Handschriften aus dem Besitz des Dietrich von Werthern und seiner Söhne in Dresden. In: Opere e giorni. Studi su mille anni di arte europea dedicati a Max Seidel. Hrsg. von Klaus Bergdolt. Venezia: Marsilio 2001. S. 371-376.

26 Vgl. Haebler, Inkunabelsammlung (wie Anm. 19); Deckert, Helmut: Katalog der Inkunabeln der Sächsischen Landesbibliothek zu Dresden. Ein Bestandsverzeichnis nach den Kriegsverlusten des Jahres 1945. Leipzig: Harrassowitz 1957, S. 14-15; Mühlner, Manfred: Inkunabelsammlung. In: Handbuch der historischen Buchbestände in Deutschland. Hrsg. von Bernhard Fabian. Bd. 17: Sachsen, A-K. Hrsg. von Friedhilde Krause. Hildesheim: Olms-Weidmann 1997. S. 140-142. http://fabian.sub.uni-goettingen.de/fabian?SL_Dresden\#2286 [Abschnitte 2.2882.292]; Mühlner, Manfred u. Frank Aurich: Inkunabelsammlung. In: Das ABC der SLUB. Lexikon der Sächsischen Landesbibliothek - Staats- und Universitätsbibliothek Dresden. Aus Anlass des 450. Gründungsjubiläums 1556-2006. Hrsg. von Thomas Bürger u. Konstantin Hermann. Dresden: Sandstein 2006. S. 115-117. http://nbn-resolving.de/urn:nbn:de:bsz:14qucosa-150144.

27 Laufer, Berthold: Verzeichnis der tibetischen Handschriften der Königlichen Bibliothek zu Dresden. In: Zeitschrift der Deutschen Morgenländischen Gesellschaft 55 (1901). S. 99-128. http://digital.slub-dresden.de/id488989973. Die handschriftlich eingetragenen Signaturen und Bemerkungen im Handexemplar der SLUB stammen eindeutig von Haebler.

28 Noch heute stecken die Handschriften in diesen Umschlägen in Schubern, jeweils mit Signaturen in Haeblers Handschrift. 
durch Aufnahme der Autografen im Dante-Apparat König Johanns von Sachsen (Mscr.Dresd.e.91) sowie der Korrespondenz in den Nachlässen Christian Hinrich Wolke (Mscr.Dresd.e.166), Johann Karl Seidemann (Mscr.Dresd.h.20) und August Wilhelm Schlegel (Mscr.Dresd.e.90) sowie der Akten der 9. Kompanie der Dresdner Kommunalgarde (Mscr.Dresd.e.175). Außerdem katalogisierte er die französischen, englischen und slawischen Handschriften (Mscr.Dresd.Oc, Od und Oe), wobei er für die tschechischen und russischen Handschriften einen Sprachkundigen hinzuziehen musste. 1904 und 1905 oblag ihm die Fortführung des Porträtkatalogs und die Eintragung der Fortsetzungen in den alphabetischen Kapselkatalog.

Die Inkunabelkatalogisierung blieb indes Haeblers Hauptarbeitsfeld. Während er 1902 noch Nachträge und Ergänzungen am Katalog der Dresdner Inkunabeln vornahm, traf er außerdienstlich bereits Vorbereitungen für eine „General-Inventarisierung“ der Inkunabeln in allen öffentlichen Bibliotheken Sachsens. Auf Anordnung der Generaldirektion der Königlichen Sammlungen unternahm er eine zehntägige Dienstreise nach Freiberg, Chemnitz, Zwickau, Annaberg und Schneeberg, um die dortigen Bestände zu sichten. 1903 ließ er sich eine größere Zahl von Miszellanbänden aus Freiberg und Zwickau zur Inventarisierung nach Dresden bringen. Auf weiteren Dienstreisen besuchte er 1904/1905 unter anderem die Bibliotheken des Gymnasiums und der JakobiKirche in Freiberg und die Bibliotheken in Zwickau und Leipzig. Von diesen Unternehmungen zeugen ein Soennecken-Briefordner mit Beschreibungen der Zwickauer, Freiberger und Oschatzer Inkunabeln sowie ein nach Hain-, Copinger- und Reichling-Nummern geordnetes Inventarregister in der SLUB. ${ }^{29}$

In den Jahren 1899 bis 1905 nahm Haebler als Dresdner Vertreter an den Versammlungen deutscher Bibliothekare in Bremen, Marburg, Gotha, Jena, Stuttgart und Posen teil. Auf dem Marburger Bibliothekartag im Jahr 1900 regte Karl Dziatzko (1842-1903) in einem Vortrag Über den Plan eines alle bekannten und noch zu ermittelnden Wiegendrucke umfassenden Katalogs an, ein ,einheitliches Verzeichnis“ der von ihm geschätzten 30000 existierenden Inkunabeldrucke „durch internationale Zusammenarbeit herzustellen“, um dem „Wert der Inkunabelkunde für bibliographische und litterarhistorische Forschung“ gerecht zu werden und ein Werkzeug für die „sichere Schätzung des Kaufwertes der Wiegendrucke“ an der Hand zu haben. ${ }^{30}$ Die Inkunabeln, die als frühe Er-

29 Vgl. Assmann, Karl: Die Sächsische Landesbibliothek von 1945 bis 1955. Zerstörung, Wiederaufbau und gegenwärtiger Stand der Arbeit. In: Sächsische Landesbibliothek Dresden 1556-1956. Festschrift zum 400-jährigen Bestehen. Leipzig: Harrassowitz 1956. S. 29-91, hier S. $58-59$.

30 Paalzow, Hans: Versammlung deutscher Bibliothekare. In: Centralblatt für Bibliothekswesen 17 (1900) H. 7. S. 337-341, hier S. 340-341. 
zeugnisse des Buchdrucks noch Merkmale handgeschriebener Codices tragen, ${ }^{31}$ wurden seit dem 17. Jahrhundert gesondert betrachtet ${ }^{32}$ und konnten im 1898 begonnenen Gesamtkatalog der Preußischen Bibliotheken allenfalls national verzeichnet, nicht aber angemessen katalogisiert werden. Hains Repertorium genügte den Ansprüchen nicht mehr. Die von Dziatzko empfohlene Kommission zur Vorbereitung des ambitionierten Unternehmens wurde auf einer Sitzung im Preußischen Kultusministerium am 28. November 1904 vom Ministerialdirektor Friedrich Althoff unter dem Namen „Kommission für den Weltkatalog der Wiegendrucke“ berufen. Sie bestand aus den Inkunabelspezialisten Ernst Voullième (Berlin), Konrad Burger (Leipzig) und Konrad Haebler, der zum Vorsitzenden und Leiter der Zentralstelle an der Königlichen Bibliothek in Berlin ernannt wurde. Außerdem konnten Adolf Schmidt (Darmstadt), Ernst Frey (München) und Isak Collijn (Uppsala) für eine Mitarbeit gewonnen werden.

Am 1. Dezember 1904 leitete Schnorr von Carolsfeld Haeblers Bitte um die letztendlich erteilte - Erlaubnis zur Ausübung seiner Funktionen beim Gesamtverzeichnis der Wiegendrucke $(\mathrm{GW})$ an die Generaldirektion der Königlichen Sammlungen mit den Worten weiter:

Ich bin der Meinung, daß der hiesigen Bibliothek aus Erfüllung der Bitte des Professors Häbler wesentliche Opfer voraussichtlich nicht erwachsen werden, wohl es ihr aber zur Ehre, möglicher Weise auch zum Vorteil gereichen wird, wenn einer ihrer Beamten bei der Ausführung eines umfassenden Werkes, das zu unternehmen die K. Preuß. Regierung als ein nobile officium für das Ursprungsland der Buchdruckerkunst betrachtet, in hervorragender Weise beteiligt sein wird. ${ }^{33}$

Wie Haeblers Jahresberichte 1905 und 1906 erkennen lassen, wurde er von seinen üblichen dienstlichen Pflichten in Dresden zunehmend entlastet und für mehrere Auslandsreisen beurlaubt. Schließlich konnte nicht ausbleiben, was im Bericht der Dresdner Bibliothek für das Jahr $1907 \mathrm{zu}$ lesen ist: „Am 1. Juni folgte Bibliothekar Prof. Dr. Häbler einem Rufe als Oberbibliothekar an der [!] Kgl. Bibliothek in Berlin." 34

31 Vgl. Geldner, Ferdinand: Inkunabelkunde. Eine Einführung in die Welt des frühesten Buchdrucks. Wiesbaden: Reichert 1978, S. 1-2.

32 Der erste selbständige Inkunabelkatalog erschien 1688 in Amsterdam (Beughem, Cornelis a: Incunabula Typographiae Sive Catalogus Librorum Scriptorumque proximis ab inventione Typographiae annis, usque ad Annum Christi M.D. inclusive, in quavis lingua editorum). Vgl. Haebler, Handbuch der Inkunabelkunde (wie Anm. 23), S. 6-7; Geldner, Inkunabelkunde (wie Anm. 31), S. 7.

33 SLUB, Bibl.Arch.III.G,Vol.623, Bl. 372r-372v.

34 SLUB, Bibl.Arch.III.G,Vol.646, Bl. 64r. Zahlreiche im Bibliotheksarchiv der SLUB erhaltene Briefe Haeblers aus Berlin an die Direktion der Dresdner Bibliothek zeigen, dass er in gutem Kontakt blieb. 
Zunächst galt es, ein verbindliches Schema zur zeitgemäßen Katalogisierung der Inkunabeln und ein Mengengerüst aufzustellen. Um die Inkunabelbestände in Deutschland zu erheben, wurden an 452 Bibliotheken Fragebögen verschickt. ${ }^{35}$ Zwischen 1906 und 1911 besuchten die Mitglieder der Kommission letztendlich 654 Bibliotheken und 22 Archive in Deutschland sowie wichtige Bibliotheken des europäischen Auslands und inventarisierten unter Mithilfe von zehn weiteren namentlich bekannten Kollegen knapp 150000 Inkunabelexemplare. ${ }^{36}$ Durch ein Netzwerk persönlicher Beziehungen konnten ähnliche nationale Inventarisierungsunternehmen im Ausland einbezogen, forciert oder angeregt werden. ${ }^{37}$ Für die sich anschließende autoptische Katalogisierung wurden Tausende Inkunabelbände nach Berlin ausgeliehen. ${ }^{38} \mathrm{Um}$ auch Inkunabeln ohne Druckvermerk bestimmen zu können, veröffentlichte Haebler 1905-1924 das bis heute unverzichtbare Typenrepertorium der Wiegendrucke, ${ }^{39}$ das er schon auf seiner Studienreise nach Spanien und Portugal 1897/98 begonnen ${ }^{40}$ und 1903 auf dem Bibliothekartag in Halle vorgestellt hatte..$^{41}$ Darin sind die für jeden Drucker charakteristischen, in Gruppen eingeteilten Formen des Leitbuchstabens M (für gotische Schriften) oder Qu (für Antiqua-Schriften) mit Proctors Maßeinheit von 20 Zeilen sowie die Form der gedruckten Initialen und Rubriken (d. h. Paragrafzeichen) zusammengestellt. Als weiteres Bestimmungshilfsmittel diente ein Tafelwerk mit Abbildungen von Beispielseiten und Typenalphabeten, das die Gesellschaft für Typenkunde ab 1907 unter Mitwirkung Haeblers in jährlichen Lieferungen herausgab..$^{42} 1910$ konnte bereits ein Probe-

35 Vgl. Haebler, Konrad: Bericht über den Plan eines Gesamtkataloges der Wiegendrucke. In: Zentralblatt für Bibliothekswesen 22 (1905) H. 11. S. 509-517; Krause, Friedhilde: Zur Geschichte des Gesamtkatalogs der Wiegendrucke. In: Zur Arbeit mit dem Gesamtkatalog der Wiegendrucke. Vorträge der Internationalen Fachtagung vom 26. bis 30. November 1979 in Berlin. Berlin: DSB 1989. S. 6-14, hier S. 7-8.

36 Vgl. Haebler, Konrad: Schlussbericht über die Inventarisierung der Inkunabeln. In: Zentralblatt für Bibliothekswesen 28 (1911) H. 7-8. S. 318-325.

37 Haebler, Wie ich Inkunabelforscher wurde (wie Anm. 22), S. 323, Anm. 36 spricht geradezu von einem Propaganda-Feldzug.

38 Vgl. Krause, Zur Geschichte des Gesamtkatalogs (wie Anm. 35), S. 11-12.

39 Haebler, Konrad: Typenrepertorium der Wiegendrucke. Bd. 1. Halle: Haupt 1907, Bd. 2-4. Leipzig: Haupt 1908-1910, 2 Ergänzungsbde. Leipzig: Harrassowitz 1922 u. 1924. Vgl. dazu http://tw.staatsbibliothek-berlin.de/.

40 Vgl. Haebler, Vom spanischen Haebler (wie Anm. 5), S. 2-3.

41 Vgl. Haebler, Konrad: Über den Plan eines Typenrepertoriums. In: Zentralblatt für Bibliothekswesen 21 (1904) H. 1-2. S. 59-73. Haeblers Dresdner Jahresbericht für 1906 ist zu entnehmen, dass er mit Unterstützung des Königlich-preußischen Kultusministeriums eine dreimonatige Auslandsreise zur Materialsammlung für das Typenrepertorium unternehmen konnte.

42 Veröffentlichungen der Gesellschaft für Typenkunde des XV. Jahrhunderts. 30 Lieferungen (= Taf. 1-2275). Leipzig [u. a.] 1907-1936. 
band des künftigen GW mit Katalogisaten von 400 bislang unbekannten Inkunabeln ${ }^{43}$ und 1914 ein beschreibendes Verzeichnis von 1570 Einblattdrucken ${ }^{44}$ erscheinen.

Am 1. Januar 1914 übernahm Haebler die Leitung der Handschriftensammlung der Königlichen Bibliothek in Berlin und wurde am 25. Dezember 1916 zum Geheimen Regierungsrat befördert. ${ }^{45}$ Nachdem das Manuskript des GW mit etwa 40000 Beschreibungen am 1. April 1920 als abgeschlossen gelten konnte, trat Haebler am 31. Dezember des folgenden Jahres in den wohlverdienten Ruhestand und kehrte zurück nach Dresden. ${ }^{46}$ Doch blieb er dort nicht untätig, sondern engagierte sich ab 1922 ehrenamtlich an der Sächsischen Landesbibliothek (SLB). So separierte er von Mai 1925 bis März 1926 unter Mithilfe der Volontärin Ilse Schunke 2100 Inkunabeln aus dem allgemeinen Druckschriftenbestand, revidierte seine bisherigen Beschreibungen, fügte Neuerwerbungen bzw. Neufunde hinzu und vergab Inkunabelsignaturen in der Reihenfolge des alphabetischchronologischen Index der Druckorte, Drucker und Drucke. ${ }^{47}$ Außerdem durchsuchte er die Altbestände nach Blinddruckeinbänden des 16. Jahrhunderts, um Abreibungen von den Rollen- und Plattenstempeln vorzunehmen. ${ }^{48}$ Das Material konnte er, wiederum mit Unterstützung von Ilse Schunke, in Form eines zweibändigen Repertoriums 1928 und 1929 publizieren. ${ }^{49}$ Als Summe seiner

43 Kommission für den Gesamtkatalog der Wiegendrucke (Hrsg.): Nachträge zu Hains Repertorium bibliographicum und seinen Fortsetzungen als Probe des Gesamtkatalogs der Wiegendrucke. Leipzig: Haupt 1910.

44 Kommission für den Gesamtkatalog der Wiegendrucke (Hrsg.): Einblattdrucke des XV. Jahrhunderts. Ein bibliographisches Verzeichnis. Halle: Karras 1914.

45 Vgl. Schmidt, Haebler (wie Anm. 2), S. 404.

46 Dort wohnte er nahe der Technischen Hochschule im Erdgeschoss des heute nicht mehr existenten Hauses Bergstraße 33 (vgl. Adressbuch für Dresden und Vororte 1926/27. Dresden 1927. Erster Teil, S. 239. http://digital.slub-dresden.de/werkansicht/dlf/72977/319/0/).

47 Vgl. Schunke, Erinnerungen an Konrad Haebler (wie Anm. 3), S. 186; Deckert, Helmut: Die übrigen Sondersammlungen. In: Sächsische Landesbibliothek Dresden 1556-1956 (wie Anm. 29). S. 166-171, hier S. 166. Die mit roter Tinte im Index und in den Beschreibungen des Haebler'schen Katalogs eingetragenen, bis heute gültigen Signaturen bestehen aus einer fortlaufenden Nummer, gefolgt von einer Formatangabe in runden Klammern. Es wurden zunächst nur geradzahlige Nummern vergeben, um Neuzugänge auf ungeraden Nummern einschieben zu können.

48 Die Abreibungen und zugehörige Zettelindizes existieren noch in der Handschriftensammlung der SLUB (Mscr.Dresd.App.309).

49 Haebler, Konrad: Rollen- und Plattenstempel des XVI. Jahrhunderts. 2 Bde. Leipzig: Harrassowitz 1928-1929. Vgl. auch Schunke, Erinnerungen an Konrad Haebler (wie Anm. 3), S. $185-187$. 
Beschäftigung mit Inkunabeln veröffentlichte Haebler 1925 sein Handbuch zur Inkunabelkunde..$^{50}$

Am 8. Oktober 1925 erschien der erste Band des GW im Verlag von Karl W. Hiersemann in Leipzig. ${ }^{51}$ Im Vorwort lobte Erich von Rath, der 1920 den Vorsitz der Kommission übernommen hatte, die „ausserordentliche Tatkraft“, mit der Haebler ,in nie ermüdender Arbeit die Grundlagen geschaffen“ habe, „auf denen das Gebäude des Katalogs errichtet werden konnte." ${ }^{\text {"52 }}$ Bis 1940 erschienen weitere sechs Bände mit jeweils einer Ergänzungs- und Verbesserungsbeilage sowie die erste Lieferung des achten Bandes (Eike von Repgow-Federicis). 1944 musste die Arbeit am GW kriegsbedingt eingestellt werden. ${ }^{53}$

Um Luftangriffen zu entgehen, zog sich Haebler 1943 als 86-jähriger kranker Mann nach Stadt Wehlen in der Sächsischen Schweiz zurück und musste von dort aus die Zerstörung seiner Heimatstadt und des Gebäudes der SLB am 13./14. Februar und am 2. März 1945 erleben. Möglicherweise erfuhr Haebler noch durch Helmut Deckert, den nachmaligen Leiter der Sondersammlungen der SLB, der ihn zu seinem 89. Geburtstag in Dorf Wehlen besuchte, ${ }^{54}$ dass der größte Teil des ihm so am Herzen gelegenen, mittlerweile auf 2269 Inkunabeln angewachsenen Dresdner Bestands durch die russische Trophäenkommission beschlagnahmt und als Kriegsbeute in die Sowjetunion abtransportiert wurde. Kurz darauf, am 13. Dezember 1946 starb der Nestor der Inkunabelkunde. In einem 1957 zum 100. Geburtstag Haeblers erschienenen Bestandsverzeichnis der verbliebenen 671 und 41 hinzugekommenen Inkunabeln der SLB resümierte Deckert, dass knapp $70 \%$ des Vorkriegsbestandes abhandengekommen waren. ${ }^{55}$

Heute, über 71 Jahre nach Haeblers Tod, haben sich handschriftliche und gedruckte Beschreibungen, Bestandsnachweise, Tafeln und Abbildungen in digitale Daten verwandelt, die über das Internet weltweit verfügbar sind. Seit Oktober 1990 wird der GW rechnergestützt bearbeitet und gedruckt. Seit August 2003 sind die nach und nach mit Normdaten angereicherten Beschreibungen der gedruckten GW-Bände sowie die sukzessive indexierten Digitalisate des noch unveröffentlichten Teils des GW-Manuskripts in einer Onlinedatenbank re-

50 Haebler, Handbuch der Inkunabelkunde (wie Anm. 23).

51 Gesamtkatalog der Wiegendrucke. Bd. 1 (Abano-Alexius). Leipzig: Hiersemann 1925.

52 Gesamtkatalog der Wiegendrucke. Bd. 1 (wie Anm. 51), S. VIII.

53 Vgl. Gesamtkatalog der Wiegendrucke. Bd. 8 (Eike von Repgow-Flühe: Nr. 9256-10101). Stuttgart: Hiersemann 1978, S. ${ }^{\star} 1$.

54 Vgl. Deckert, Katalog der Inkunabeln (wie Anm. 26), S. 12.

55 Vgl. Deckert, Katalog der Inkunabeln (wie Anm. 26), S. 10. Zwischen Inkunabelausgaben oder Inkunabelexemplaren wird hier und in der übrigen Literatur zu den Dresdner Inkunabeln nicht klar unterschieden. 
cherchierbar. ${ }^{56}$ Die Bearbeitung wird in alphabetischer Reihenfolge der Verfassernamen und Anonyma fortgeführt, die Ergebnisse werden weiterhin lieferungsweise gedruckt. ${ }^{57}$ In der GW-Datenbank sind momentan 1285 Inkunabelausgaben der SLUB einschließlich der heute in der Russischen Staatsbibliothek in Moskau befindlichen erfasst. Der GW ist verlinkt mit dem seit 2003 online verfügbaren Incunable Short Title Catalogue (ISTC), der ergänzend zum GW dem Nachweis der etwa 500000 weltweit vorhandenen Inkunabelexemplare dient. ${ }^{58}$ Dort werden für die SLUB bislang 1089 Exemplare $^{59}$ und davon 494 nach Moskau verlagerte Exemplare ${ }^{60}$ angezeigt. Im Inkunabelkatalog der Russischen Staatsbibliothek ${ }^{61}$ findet man rund 1500 Inkunabeln mit der Provenienz „Dresden Sächsische Landesbibliothek" und den Dresdner Signaturen. ${ }^{62}$ Der seit 2000 online zugängliche, mit dem GW und dem ISTC verknüpfte Inkunabelkatalog deutscher Bibliotheken (INKA) ${ }^{63}$ weist für die SLUB den aktuellen Bestand von 806 Inkunabelausgaben und 840 Exemplaren mit Signaturen nach. ${ }^{64}$

56 Vgl. http://www.gesamtkatalogderwiegendrucke.de; Generaldirektor der Staatsbibliothek zu Berlin, Preußischer Kulturbesitz (Hrsg.): Berichte zur Geschichte der Deutschen Staatsbibliothek in Berlin. Wiesbaden: Reichert 1996. S. 125-126 u. 127-128; Suckow, Ninon u. Werner Klarkowski: Die Datenbank „Gesamtkatalog der Wiegendrucke“. In: Zeitschrift für Bibliothekswesen und Bibliographie 51 (2004) H. 4. S. 200-206. http://zfbb.thulb.uni-jena.de/servlets/ MCRFileNodeServlet/jportal_derivate_00001691/j04-h4-auf-1.pdf.

57 Zuletzt erschien die zweite Lieferung von Bd. 12: Abkürzungen für Wiegendrucksammlungen (Sigelliste; Fortsetzung); Nachträge und Berichtigungen der Sigelliste; De horis canonicis dicendis - Hugo Senensis (Nummer 13538-13646). Stuttgart: Hiersemann 2016.

58 Vgl. Horn, Reinhard u. Gertrud Friedl: Der bundesdeutsche Inkunabel-Census. Ein Teil des Incunable Short Title Catalogue (ISTC). In: Zeitschrift für Bibliothekswesen und Bibliographie 38 (1991) H. 4. S. 368-383; Wagner, Bettina: Die ältesten Drucke im Internet. Vom lokalen Inkunabelkatalog zu einem koordinierten nationalen Digitalisierungsprojekt. In: Zeitschrift für Bibliothekswesen und Bibliographie 58 (2011) H. 1. S. 19-27. http://zfbb.thulb.uni-jena.de/servlets/MCRFileNodeServlet/jportal_derivate_00202174/j11-h1-auf-3.pdf.

$59 \mathrm{Vgl}$. http://data.cerl.org/istc/_search?query=\%22Dresden $\% 20$ SLUB $\% 22 \% 20 \% 20$ AND $\%$ 20data.holdings.holding_institution_name.orig:\%22Dresden $\% 20$ SLUB $\% 22 \&$ from $=0$.

60 Vgl. http://data.cerl.org/istc/_search?query=\%22Dresden\%20SLUB\%22\%20AND\%20data. holdings.holding_institution_name.orig:\%22Moscow\%20SL\%22\&from $=0$.

$61 \mathrm{Vgl}$. http://aleph.rsl.ru/F/?func=file\&file_name=find-b\&local_base=xink\&CON_LNG=ENG. Die Signaturen werden nur im MARC-Format angezeigt.

62 Das entspricht in etwa der von Mühlner, Inkunabelsammlung (wie Anm. 26), S. 140 angegebenen Zahl von 1539 verlagerten Inkunabeln.

63 Vgl. dazu Mehringer, Ulrike und Armin Schlechter: Der Inkunabel-Katalog deutscher Bibliotheken (INKA). In: b.i.t.online 5 (2002) H. 1. S. 41-44. http://www.b-i-t-online.de/archiv/200201/nach1.htm.

64 Vgl. http://www.inka.uni-tuebingen.de/cgi-bin/inkunabel?sbibliothek=dre\&form=voll\&stitel $=\&$ sperson $=\&$ stkz $=$ jede\&sort $=\&$ sbubi $=\&$ sdrucker $=\&$ sprovenien $z=\&$ sjahr $=\&$ ssignatur $=\& s-$ nachweis $=\&$ naw $=$ GW\&skyriss $=\&$ snummer $=\&$ sinkanum $=\&$ searchs $=\&$ digi $=\#$ anfang . 
Wie auch die Titel der UB Heidelberg und der UB Leipzig sind die Dresdner INKA-Daten inzwischen in den Südwestdeutschen Bibliotheksverbund eingespielt und werden mit den exemplarspezifischen Daten aus dem Inkunabelkatalog von Deckert angereichert.

Wäre Haebler als „Pionier der Großunternehmungen“65 noch am Leben, hätten ihn diese nationalen und internationalen Datenbanken gewiss ebenso begeistert wie die zunehmende Digitalisierung von Inkunabeln mit Nachweis in GW und ISTC. Die Zahl der momentan in den Digitalen Sammlungen der SLUB vorhandenen 18 Inkunabeln ${ }^{66}$ wird sich dank des Sächsischen Landesdigitalisierungsprogrammes bald um zahlreiche weitere weltweit noch nicht digitalisierte Titel erhöhen. 